Research Article

\title{
Coupled Fixed Point Theorems for Some Type of Contraction Mappings in $b$-Cone and $b$-Theta Cone Metric Spaces
}

\author{
Sahar Mohamed Ali Abou Bakr \\ Department of Mathematics, Faculty of Science, Ain Shams University, Cairo, Egypt \\ Correspondence should be addressed to Sahar Mohamed Ali Abou Bakr; saharm_ali@yahoo.com
}

Received 15 February 2021; Revised 27 March 2021; Accepted 17 April 2021; Published 3 May 2021

Academic Editor: Ching-Feng Wen

Copyright (C) 2021 Sahar Mohamed Ali Abou Bakr. This is an open access article distributed under the Creative Commons Attribution License, which permits unrestricted use, distribution, and reproduction in any medium, provided the original work is properly cited.

This paper proves the existence of a unique coupled fixed point of some type of contraction mappings defined on a complete $b$-cone and $b$-theta cone metric spaces; consequently, it extends and generalizes many previous coupled fixed point theorems.

\section{Introduction}

In 2007, Huang and Zhang [1] introduced cone metric spaces as generalization of metric spaces by considering vector-valued metrics with values in an ordered real Banach space and hence generalized the concept of metric spaces and its completeness. They proved the existence of a unique fixed point for a contraction self-map $T$ of cone metric space $(X, d)$ showing that cone metric spaces provide larger categories of spaces for the fixed point theory. Recall that if $\mathbb{A}$ is a normed space, $C$ is a cone in $\mathbb{A}$ that generates the partial ordered relation $\preceq$, and $(X, d)$ is a cone metric space over $\mathbb{A}$, then a mapping $T: X \longrightarrow X$ is said to be contraction on $X$ if and only if there is a constant $\alpha \in[0,1)$ such that

$$
d(T(x), T(y)) \preceq \alpha d(x, y), \quad \forall x, y \in X .
$$

In 2008, Rezapour and Hamlbarani [2] improved some of the results in [1] by omitting the normality assumption of the cone induced the partial relation.

After that, more topological characterization of cone metric spaces linked with some fixed point theorems have been studied by many other authors (see [2-5] and the references therein).

In 2013, 2014, 2016, 2017, and then in 2020, Azam et al., citeAkbar, Xu and Radenovi [6], Huang and Radenovi [7], Sharma [8], and Sahar [9], respectively, considered cyclic and cone metric spaces over Banach algebra and $b$ - cone metric spaces as a generalization of cone metric spaces, and they gave some further generalizations of some fixed points and proved fixed point theorems of contractive mapping in $b$ - cone metric spaces, some of these results are proved without using the normality condition of a cone and some proved for generalized contraction multivalued mappings.

In 2020, Sahar [10] introduced the concept of theta cone metric spaces which gave larger categories of metric spaces and generalized some previous fixed points' theorems in the setting of this concept.

On the other side, in 1987, the concept of coupled fixed point was initiated by Gue and Lakshmikantham [11], in partially ordered metric spaces; after that, in 2006, Bhaskar and Lakshmikantham [12] proved existence of coupled fixed points for mappings having the mixed monotone property.

In 2009, Sabetghadam et al. [13] proved some coupled fixed point theorems for mappings satisfying different contractive conditions on complete cone metric spaces. Specifically, they proved the following.

Theorem 1 (see [13]). Let $(X, C d)$ be a complete cone metric space. Suppose that the mapping $F: X \times X \longrightarrow X$ satisfies the following contractive condition for all $x, y, u, v \in X$ :

$$
d(F(x, y), F(u, v)) \preceq k d(x, u)+l d(y, v),
$$


where $k$ and $l$ are nonnegative constants with $k+l<1$. Then, $F$ has a unique coupled fixed point.

In 2010, Khamsi [14] gave the definition of metric type space (or $b$-metric space) and used this approach to proved the existence of a unique fixed point for Lipschitzian type mapping defined on a complete metric type space, and then, he noticed that, in case of normal cone, the cone metric $q$ of the cone metric space $(X, C, q)$ generates a metric type $D: X \times$ $X \longrightarrow \mathbb{R}^{+}$and hence a metric type space $(X, D)$. His remarkable notice enabled him to prove the existence of unique fixed point for Lipschitzian type mapping defined on complete cone metric $(X, C, q)$ but provided that the cone $C$ is normal.

In 2013, Luong et al. [15] followed another direction; they proved some coincidence and coupled fixed point of two compatible mappings: $F: X \times X \longrightarrow X$ and $g: X \longrightarrow X, F$ is continuous, $F$ has the mixed $g$-monotone property, $F$ and $g$ satisfy some generalized contraction conditions, $X$ is partially ordered set endowed with a complete cone metric and some extra conditions on convergent monotone nondecreasing and convergent monotone nonincreasing sequences in $X$.

In 2021, Sahar [16] generalized coupled fixed point result for some generalized contraction type of mappings of Bhaskar and Lakshmikantham [12] and of Sahar [17, 18] in theta cone metric spaces.

We have the following notations and basic definitions.

\section{Preliminaries and Basic Definitions}

We start with the following.

Definition 1 (see [14]). Let $X$ be a nonempty set. Let $D: X \times$ $X \longrightarrow \mathbb{R}^{+}$be a function which satisfies the following:

(1) $D(x, y) \geq 0, D(x, y)=0$ if and only if $x=y$

(2) $D(x, y)=D(y, x)$ for every $x, y \in X$

(3) $D(x, y) \leq K[D(x, w)+D(w, y)]$ for some positive real numberK $>0$

The pair $(X, D)$ is called a metric type space, and it is called $b$-metric space if $K \geq 1$.

We recall some standard notations and definitions in cone metric spaces.

A subset $C$ of a linear space $A$ is said to be a cone in $A$ if and only if

(1) $C$ is nonempty closed and $C \neq\{\theta\}$, where $\theta$ is the zero (neutral element) of $A$

(2) $\lambda C+\mu C \subset C$ for all nonnegative real numbers $\lambda, \mu$

(3) $C \cap-C=\{\theta\}$

A cone $C$ in a normed space $(\mathbb{A},\|\cdot\|)$ is said to be solid if and only if it has a nonempty interior, that is, the set of all interior points of $C$ is not empty set, $\operatorname{Int} C \neq \varnothing$.

If $(\mathbb{A},\|\cdot\|)$ is a normed space, $C$ is a cone in $\mathbb{A}$; then, $C$ generates the following ordered relations:

$$
\begin{aligned}
& u \leq v \Longleftrightarrow v-u \in C, \\
& u<v \Longleftrightarrow(v-u \in C \text { and } u \neq v), \\
& u \ll v \Longleftrightarrow v-u \in \operatorname{Int} C .
\end{aligned}
$$

A sequence $\left\{z_{n}\right\}_{n \in N}$ in $\mathbb{A}$ is bounded above by $z \in \mathbb{A}$ if and only if

$$
z_{n} \preceq z, \quad \forall n \in \mathbb{N},
$$

and its bounded below by $z \in \mathbb{A}$ if and only if

$$
z \preceq z_{n}, \quad \forall n \in \mathbb{N} .
$$

A cone $C$ is called normal if there is a number $M>0$ (later proved to be greater than or equal 1) such that, for all $x, y \in \mathbb{A}$,

$$
\text { if } \theta \leq x \leq y, \quad \text { then }\|x\| \leq M\|y\| .
$$

The normal constant of the normal cone $C$ is defined to be the smallest constant $M$ satisfying (6).

A cone $C$ is called regular if every monotonically nonincreasing (no-decreasing) bounded above (bounded below) sequence has a limit in the norm sense of $\mathbb{A}$.

Remark 1 (see [3]). Every regular cone is normal, there are normal cones which are not regular, there are cones which are not normal, the normal constant $M$ of any normal cone is such that $M \geq 1$, and for any real number $k, k \geq 1$, there is a cone with normal constant $M=k$.

Definition 2. Suppose that $X$ is a nonempty set, $C$ is a cone in a normed space $\mathbb{A}, r \in \mathbb{R}^{+}, r \geq 1$ (this $r$ does not depend on the cone $C$ itself, the cone may not be normal), and $q$ is a function; $q: X \times X \longrightarrow C$ satisfies the following:

(1) $\theta \ll q(u, v), \forall u, v \in X$

(2) $q(u, v)=\theta \Leftrightarrow u=v$

(3) $q(u, v)=q(v, u), \quad \forall u, v \in X$

(4) $q(u, v) \leq r[q(u, w)+q(w, v)], \quad \forall u, v, w \in X$

Then, $(X, C, q)$ is defined to be a $b$-cone metric space over $C$. In particular, if $r=1$, then $(X, C, q)$ is cone metric space.

A sequence $\left\{v_{n}\right\}_{n \in \mathbb{N}}$ in a cone (b-cone) metric space $(X, C, q)$ (with $C$ solid) is Cauchy if and only if for every $z \in \mathbb{A}$ with $\theta \ll z$ there is $n_{0} \in \mathbb{N}$ such that $\mathfrak{q}\left(v_{n}, v_{m}\right) \ll z$ for all $n, m \geq n_{0}$.

A sequence $\left\{v_{n}\right\}_{n \in \mathbb{N}}$ in a cone (b-cone) metric space $(X, C, q)$ (with $C$ solid) is convergent sequence if and only if there is $v$ such that, for every $z \in \mathbb{A}$ with $\theta \ll z$, there is $n_{0} \in \mathbb{N}$ such that $q\left(v_{n}, v\right) \ll z$ for all $n \geq n_{0}$.

A cone $(b$ - cone) metric space $(X, C, q)$ is complete whenever every Cauchy sequence in $(X, C, q)$ converges to an element belonging to $(X, C, q)$.

The following is a remarkable and an excitable notice given by Khamsi [14].

Remark 2. If $(X, C, q)$ is a cone metric space, where $C$ is normal cone with normal constant $M \geq 1$, then the composite function $D(x, y)=(\|\cdot\| o q)(x, y)=\|q(x, y)\|$, $D: X \times X \longrightarrow \mathbb{R}^{+}$is a $b$-metric on $X$. Indeed, for all $x, y, w \in X$, we have 
(1) $D(x, y) \geq 0$ and $D(x, y)=0$ if and only if $\|q(x, y)\|=0$, if and only if $q(x, y)=\theta$, and if and only if $x=y$

(2) $D(x, y)=\|q(x, y)\|=\|q(y, x)\|=D(y, x)$

(3) Since $q(x, y) \leq q(x, w)+q(w, y)$, then $\|q(x, y)\| \leq$ $M[\|q(x, w)+q(w, y)\|] ;$ hence, $\quad D(x, y)=\| q(x$, $y) \| \leq M[\|q(x, w)+q(w, y)\|] \leq \quad M[\|q(x, w)\|+$ $\|q(w, y)\|]=M[D(x, w)+D(w, y)]$

That is, the cone metric $(X, C, q)$ for normal cone $C$ generates a $b$-metric space $(X, D)$.

Next, we have the following.

Definition 3 (see [10]). Let be an ordered linear space, where $\preceq$ is an ordered relation induced by some cone $C \subset \mathbb{A}$ and $\Theta: C \times C \longrightarrow C$ be a continuous mapping with respect to each variable, and we denote

$$
\operatorname{Im}(\Theta)=\left\{t: t \in C \text { such that } \exists u_{0}, v_{0} \in \mathbb{A}, \Theta\left(u_{0}, v_{0}\right)=t\right\} .
$$

Then, $\Theta$ is said to be an ordered-action mapping on $\mathbb{A}$ if and only if it satisfies the following conditions:

(1) $\Theta(\theta, \theta)=\theta$ and $\Theta(u, v)=\Theta(v, u)$, for every $u, v \in C$

(2) $\Theta(u, v)<\Theta(w, t)$ if either $\left\{\begin{array}{l}u<w \text { and } v \preceq t \\ \text { or } \\ u \preceq w \text { and } v<t\end{array}\right.$

(3) For every $u \in \operatorname{Im}(\Theta)$ and every $\theta \preceq v \preceq u$, there is $\theta \preceq w \preceq u$ such that $\Theta(v, w)=u$

(4) $\Theta(u, \theta) \preceq u$, for every $u \in C /\{\theta\}$ follows.

In addition, the concept of $\Theta$-cone-metric space is as

Definition 4 (see $[10])$. Let $(\mathbb{A}, \preceq)$ be an ordered normed space, where $\preceq$ is an ordered relation induced by some cone $C \subset \mathbb{A}$ and $\Theta$ be an ordered-action mapping on $\mathbb{A}$. If $X$ is a nonempty set, then a function $d_{\Theta}: X \times X \longrightarrow C$ is said to be $\Theta$-cone-metric on $X$ if and only if $d_{\Theta}$ satisfies the following conditions:

(1) $d_{\Theta}(x, y)=\theta \Leftrightarrow x=y$

(2) $d_{\Theta}(x, y)=d_{\Theta}(y, x), \forall x, y \in X$

(3) $d_{\Theta}(x, y) \preceq r \Theta\left(d_{\Theta}(x, z), d_{\Theta}(z, y)\right), \forall x, y, z \in X$, where $r$ is real number such that $r \geq 1$

The triple $\left(X, C, d_{\Theta}\right)$ is defined to be a $b$ - $\Theta$-cone-metric space.

Remark 3. The constant $r$ does not depend on the cone $C$ in general whether it is normal cone or not.

If $r=1$, then $\left(X, C, d_{\Theta}\right)$ is $\Theta$-cone-metric space, meaning that the class of all $\Theta$-cone-metric spaces is included in the class of all $b$ - $\Theta$-cone-metric spaces.

If $\Theta(u, v)=u+v$, then $\left(X, C, d_{\Theta}\right)$ is $b$ - cone-metric space, meaning that the class of all cone metric spaces is included in the class of all theta cone metric spaces.

A sequence $\left\{u_{n}\right\}_{n \in \mathbb{N}}$ in $\left(X, C, d_{\Theta}\right)$ converges to $u$ whenever, for each $z \in \operatorname{Im}(\theta)$ with $\theta<z$, there is $n_{0} \in \mathbb{N}$ such that $d_{\Theta}\left(u_{n}, u\right)<z$ for all $n \geq n_{0}$. We instead write $u_{n} \longrightarrow \stackrel{a_{\Theta}}{\longrightarrow} \infty u$.

A sequence $\left\{u_{n}\right\}_{n \in \mathbb{N}}$ in $\left(X, C, d_{\Theta}\right)$ is Cauchy whenever, for each $z \in \operatorname{Im}(\theta)$ with $\theta<z$, there is $n_{0} \in \mathbb{N}$ such that $d_{\Theta}\left(u_{n}, u_{m}\right)<z$ for all $n, m \geq n_{0}$.

$\mathrm{A} \Theta$ cone $\left(b\right.$ - cone) metric space $\left(X, C, d_{\Theta}\right)$ is complete whenever every Cauchy sequence in $\left(X, C, d_{\Theta}\right)$ converges to an element belonging to $\left(X, C, d_{\Theta}\right)$.

On the other side, we have the following.

Definition 5. An element $(x, y) \in X \times X$ is said to be a coupled fixed point of the mapping $F: X \times X \longrightarrow X$ if and only if $F(x, y)=x$ and $F(y, x)=y$.

In this paper, we consider the corresponding definition for contraction type of mappings on complete $b$-cone metric spaces and generalize the coupled fixed point theorem of Sabetghadam et al. (Theorem 1) in this setting. On the other side, we consider the concept of $b$-theta-cone metrics and also prove the existence of unique coupled fixed point of some contraction type of mappings that gives another generalization of some previous coupled fixed point theorems.

Since the class of all $b$-cone metric spaces is including the class of cone metric spaces as supported with the example below, the results of this paper are real generalizations of some previous results. Moreover and in particular, some results of [15] can be extended to the case of theta cone metric spaces and do not affect the validity of this paper.

\section{Main Results}

Let $p$ be any real number, $p>0$, and $C_{p}:=\left\{\left\{\lambda_{n}\right\}_{n \in \mathbb{N}}\right.$ : $\left.\left\{\lambda_{n}\right\}_{n \in \mathbb{N}} \in l_{p}, \lambda_{n} \geq 0 \forall n \in \mathbb{N}\right\}$. Then, $C_{p}$ is a normal cone in the Banach space $l_{p}$ with normal constant $M=1$, where $\left\|\left\{\lambda_{n}\right\}_{n \in \mathbb{N}}\right\|_{p}=\sqrt[p]{\sum_{n \in \mathbb{N}}\left|\lambda_{n}\right|^{p}} \quad$ for every $\quad\left\{\lambda_{n}\right\}_{n \in \mathbb{N}} \in l_{p}$, if $\left\{\lambda_{n}\right\}_{n \in \mathbb{N}} \preceq\left\{\mu_{n}\right\}_{n \in \mathbb{N}}$, then $\left\{\mu_{n}-\lambda_{n}\right\}_{n \in \mathbb{N}} \in C_{p}$, meaning that $\mu_{n}-\lambda_{n} \geq 0, \quad \lambda_{n} \leq \mu_{n}$ for every $n \in \mathbb{N}$ and therefore $\sqrt[p]{\sum_{n \in \mathbb{N}}\left|\lambda_{n}\right|^{p}} \leq \sqrt[p]{\sum_{n \in \mathbb{N}}\left|\mu_{n}\right|^{p}}, \quad$ equivalently, $\quad\left\|\left\{\lambda_{n}\right\}_{n \in \mathbb{N}}\right\|_{p} \leq$ $\left\|\left\{\mu_{n}\right\}_{n \in \mathbb{N}}\right\|_{p}$.

The class of cone metric spaces is larger than the class of metric spaces. Indeed, we have the following.

From any metric space $(X, d)$, we define infinitely many cone metric spaces in such a way that if $\left(X, C, d^{*}\right)$ is one of these cone metric spaces, then we have

$$
d(u, v)=\left\|d^{*}(u, v)\right\|, \quad \forall u, v \in X .
$$

Indeed, let $(X, d)$ be a metric space, $p$ be a given real number, and $\lambda=\left\{\lambda_{n}\right\}_{n \in \mathbb{N}} \in l_{p}$. Define

$$
d^{*}(u, v)=\left\{\frac{d(u, v)\left|\lambda_{n}\right|}{\|\lambda\|_{p}}\right\}_{n \in \mathbb{N}}, \quad \forall u, v \in X .
$$

Clearly, $d^{*}: X \times X \longrightarrow C_{p}$ is cone metric on $X$ and $d(u, v)=\left\|d^{*}(u, v)\right\|_{p}$ for every $u, v \in X$.

Specifically and in particular, the following is cone metric on $X$ and $d(u, v)=\left\|d^{*}(u, v)\right\|_{p}$ for every $u, v \in X$ : 


$$
d^{*}(u, v)=\left\{\frac{d(u, v)}{2^{(n / p)}}\right\}_{n \in \mathbb{N}}, \quad \forall u, v \in X
$$

Every cone metric space is $b$-cone metric space with $r=$ 1 and the converse is not true, meaning that the class of all $b$-cone metric spaces is larger than the class of all cone metric spaces. Indeed, we have the following.

Let $M_{m}\left(l_{p}\right)$ be the Banach space of all $m \times m$ matrices, and the entries of each matrix in $M_{m}\left(l_{p}\right)$ are elements of $l_{p}$, the usual linear structure of addition and scalar multiplication:

$$
\begin{aligned}
A & =\left[a^{i j}\right]_{1 \leq i, j \leq m} \in M_{m}\left(l_{p}\right) \Longrightarrow a^{i j}=\left\{a_{n}^{i j}\right\}_{n \in \mathbb{N}} \in l_{p}, \quad \forall 1 \leq i, j \leq m, \\
\|A\|_{\infty} & =\left\|\left[a^{i j}\right]_{1 \leq i, j \leq m}\right\|_{\infty}=\max _{i=1}^{m} \sum_{j=1}^{m}\left\|a_{i j}\right\|_{p} .
\end{aligned}
$$

Let $C^{*}:=\left\{\left[a^{i j}\right]_{1 \leq i, j \leq m}: a^{i j} \in C_{p}, \forall 1 \leq i, j \leq m\right\}$. Then, $C^{*}$ is a normal cone in $\mathbb{A}=M_{m}\left(l_{p}\right)$ with normal constant $M=1$.

Let us now give an example of $b$-cone metric space which is not cone metric space.

Let $X=M_{m}\left(l_{p}\right)$, using the fact that the cone is normal with normal constant $M=1$, and Khamsi notices that every cone metric $p$ on $X$ generates a metric $D(x, y)=\|p(x, y)\|$ on $X, D: X \times X \longrightarrow \mathbb{R}^{+}$.

Differently, we have the following. Let $p$ be a real number, $0<p<1$, and $q, q: X \times X \longrightarrow C^{*}$ be defined by

$$
\begin{aligned}
q(A, B) & =q\left(\left[a^{i j}\right]_{1 \leq i, j \leq m},\left[b^{i j}\right]_{1 \leq i, j \leq m}\right) \\
& :=\left[\left\{\left\|a^{i j}-b^{i j}\right\|_{p} \delta_{j k}\right\}_{k \in \mathbb{N}}\right]_{1 \leq i, j \leq m} .
\end{aligned}
$$

Then, $\left(X, C^{*}, q\right)$ is $b$-cone metric space with $r=2^{(1 / p)}$ which is not a cone metric space. Indeed, let $A, B, C \in X$ with $A=\left[a^{i j}\right]_{1 \leq i, j \leq m}, B=\left[b^{i j}\right]_{1 \leq i, j \leq m}$, and $C=\left[c^{i j}\right]_{1 \leq i, j \leq m}$, and we have

$$
\begin{aligned}
q(A, B) & =q\left(\left[a^{i j}\right]_{1 \leq i, j \leq m},\left[b^{i j}\right]_{1 \leq i, j \leq m}\right) \\
& =\left[\left\{\left\|a^{i j}-b^{i j}\right\|_{p} \delta_{j k}\right\}_{k \in \mathbb{N}}\right]_{1 \leq i, j \leq m}=\left[\left\{\left\|\left(a^{i j}-c^{i j} t+n q h c^{i j}-b^{i j}\right)\right\|_{p} \delta_{j k}\right\}_{k \in \mathbb{N}}\right]_{1 \leq i, j \leq m} \\
& \prec\left[\left\{2^{(1 / p)}\left(\left\|a^{i j}-c^{i j}\right\|_{p}+\left\|c^{i j}-b^{i j}\right\|_{p}\right) \delta_{j k}\right\}_{k \in \mathbb{N}}\right]_{1 \leq i, j \leq m} \\
& =2^{(1 / p)}\left[\left\{\left\|a^{i j}-c^{i j}\right\|_{p} \delta_{j k}\right\}_{k \in \mathbb{N}}+\left\{\left\|c^{i j}-b^{i j}\right\|_{p} \delta_{j k}\right\}_{k \in \mathbb{N}}\right]_{1 \leq i, j \leq m} \\
& =2^{(1 / p)}\left(\left[\left\{\left\|a^{i j}-c^{i j}\right\|_{p} \delta_{j k}\right\}_{k \in \mathbb{N}}\right]_{1 \leq i, j \leq m}+\left[\left\{\left\|c^{i j}-b^{i j}\right\|_{p} \delta_{j k}\right\}_{k \in \mathbb{N}}\right]_{1 \leq i, j \leq m}\right) \\
& =2^{(1 / p)}(q(A, C)+q(C, B)) .
\end{aligned}
$$

Here, the constant $r=2^{(1 / p)}$ is completely different from the normal constant of the cone which is $M=1$ in addition that the range of $q$ is a subset of the given cone not subset of positive real numbers $\mathbb{R}^{+}$. That is why the results of this paper do not interfere, but it provides generalized results of [14].

We have the following results, where cones are not necessarily normal but solid.

Theorem 2. Let $(X, C, q)$ be a complete b-cone metric space. Suppose that the mapping $T: X \times X \longrightarrow X$ satisfies the following contractive condition:

$$
q(T(x, y), T(u, v)) \preceq k q(x, u)+l q(y, v), \quad \forall x, y, u, v \in X,
$$

where $k$ and $l$ are nonnegative constants with $r(k+l)<1$. Then, $T$ has a unique coupled fixed point.

Proof. Select $u_{0}, v_{0}$ in $X$ and set $u_{1}=T\left(u_{0}, v_{0}\right), v_{1}=$ $T\left(v_{0}, u_{0}\right), \ldots, u_{n+1}=T\left(u_{n}, v_{n}\right), v_{n+1}=T\left(v_{n}, u_{n}\right)$. Then, by (14), we have

$$
\begin{aligned}
q\left(u_{n+1}, u_{n}\right) & =q\left(T\left(u_{n}, v_{n}\right), T\left(u_{n-1}, v_{n-1}\right)\right) \\
& \preceq k q\left(u_{n}, u_{n-1}\right)+l q\left(v_{n}, v_{n-1}\right) .
\end{aligned}
$$

Similarly,

$$
\begin{aligned}
q\left(v_{n+1}, v_{n}\right) & =q\left(T\left(v_{n}, u_{n}\right), T\left(v_{n-1}, u_{n-1}\right)\right) \\
& \preceq k q\left(v_{n}, v_{n-1}\right)+l q\left(u_{n}, u_{n-1}\right) .
\end{aligned}
$$

Let $z_{n}=q\left(v_{n}, v_{n-1}\right)+q\left(u_{n}, u_{n-1}\right)$. Then, we have 


$$
\begin{aligned}
z_{n+1} & =q\left(u_{n+1}, u_{n}\right)+q\left(v_{n+1}, v_{n}\right) \\
& \leq k q\left(u_{n}, u_{n-1}\right)+l q\left(v_{n}, v_{n-1}\right)+k q\left(v_{n}, v_{n-1}\right)+l q\left(u_{n}, u_{n-1}\right) \\
& =(k+l)\left[q\left(u_{n}, u_{n-1}\right)+q\left(v_{n}, v_{n-1}\right)\right]=(k+l) z_{n} .
\end{aligned}
$$

For each $n \in \mathbb{N}$, we have

$$
\theta \preceq z_{n} \preceq(k+l) z_{n-1} \preceq(k+l)^{2} z_{n-2} \preceq, \ldots, \preceq(k+l)^{n} z_{0}
$$

If $z_{0}=\theta$, then $\left(x_{0}, y_{0}\right)$ is coupled fixed point of $T$; therefore, we continue by letting $\theta<z_{0}$. Now, let $n, m \in \mathbb{N}$, $n \leq m$. Then,

$$
\begin{aligned}
& q\left(u_{n}, u_{m}\right) \leq r\left[q\left(u_{n}, u_{n+1}\right)+q\left(u_{n+1}, u_{m}\right)\right] \\
& \leq r\left[q\left(u_{n}, u_{n+1}\right)+r\left[q\left(u_{n+1}, u_{n+2}\right)+q\left(u_{n+2}, u_{m}\right)\right]\right] \\
& \leq r\left[q\left(u_{n}, u_{n+1}\right)+r\left[q\left(u_{n+1}, u_{n+2}\right)+r\left[q\left(u_{n+2}, u_{n+3}\right)+q\left(u_{n+3}, u_{m}\right)\right]\right]\right] \\
&= r q\left(u_{n}, u_{n+1}\right)+r^{2} q\left(u_{n+1}, u_{n+2}\right)+r^{3} q\left(u_{n+2}, u_{n+3}\right)+\cdots \cdots \\
&+r^{m-n} q\left(u_{m-1}, u_{m}\right) .
\end{aligned}
$$

Similarly,

$$
q\left(v_{n}, v_{m}\right) \preceq r q\left(v_{n}, v_{n+1}\right)+r^{2} q\left(v_{n+1}, v_{n+2}\right)+r^{3} q\left(v_{n+2}, v_{n+3}\right)+\cdots \cdots+r^{m-n} q\left(v_{m-1}, v_{m}\right) .
$$

Adding, we obtain

$$
\begin{aligned}
& q\left(u_{n}, u_{m}\right)+q\left(v_{n}, v_{m}\right) \preceq r\left[q\left(u_{n}, u_{n+1}\right)+q\left(v_{n}, v_{n+1}\right)\right]+ \\
& \quad+r^{2}\left[q\left(u_{n+1}, u_{n+2}\right)+q\left(v_{n+1}, v_{n+2}\right)\right]+ \\
& \quad+r^{3}\left[q\left(u_{n+2}, u_{n+3}\right)+q\left(v_{n+2}, v_{n+3}\right)\right]+\cdots+r^{m-n}\left[q\left(u_{m-1}, u_{m}\right)+q\left(v_{m-1}, v_{m}\right)\right] .
\end{aligned}
$$

This shows that

$q\left(u_{n}, u_{m}\right)+q\left(v_{n}, v_{m}\right) \preceq r z_{n+1}+r^{2} z_{n+2}+r^{3} z_{n+3}+\cdots+r^{m-n} z_{m}$.

$$
\begin{aligned}
q\left(u_{n}, u_{m}\right)+q\left(v_{n}, v_{m}\right) \preceq r(k+l)^{n+1} z_{0}+r^{2}(k+l)^{n+2} z_{0} \\
\quad+\cdots+r^{m-n}(k+l)^{n+(m-n)} z_{0} \\
=(k+l)^{n}\left[r(k+l)+r^{2}(k+l)^{2}+r^{3}(k+l)^{3}+\cdots+r^{m-n}(k+l)^{m-n}\right] z_{0} \\
=(k+l)^{n}\left[[r(k+l)]+[r(k+l)]^{2}+[r(k+l)]^{3}+\cdots+[r(k+l)]^{m-n}\right] z_{0} \\
\preceq(k+l)^{n}\left[\frac{r(k+l)}{1-[r(k+l)]}\right] z_{0} .
\end{aligned}
$$

Let $\theta \ll z$, that is, $z \in \operatorname{Int}(C)$. Then, there is a neighborhood of $z$ with some radius $\delta>0$ say $z+N_{\delta}(\theta)$ such that
$z+N_{\delta}(\theta) \subset C$, and for this $\delta>0$, there is a natural number $n_{0}$ such that $(k+l)^{n}<\delta\left[\left(1-[r(k+l)] /\left\|z_{0}\right\| r(k+l)\right)\right]$ for 
every $n \geq n_{0}$; hence, $\left[\left(\left\|z_{0}\right\| r(k+l) / 1-[r(k+l)]\right)\right](k+l)^{n}<\delta$ for every $n \geq n_{0}$; consequently, $\quad[\|(r(k+l) / 1-[r(k+$ l)] $\left.\|(k+l)^{n} z_{0}\right]<\delta$ for every $n \geq n_{0}$. This proves that $\pm(r(k+$ $l) / 1-[r(k+l)])(k+l)^{n} z_{0} \in N_{\delta}(\theta)$ for every $n \geq n_{0}$. Therefore, $z \pm(r(k+l) / 1-[r(k+l)])(k+l)^{n} z_{0} \in z+N_{\delta}(\theta) \subset C$; hence, $z \pm(r(k+l) / 1-[r(k+l)])(k+l)^{n} z_{0} \in \operatorname{Int}(C)$ for every $n \geq n_{0}$ :

$$
\frac{r(k+l)}{1-[r(k+l)]}(k+l)^{n} z_{0} \ll z, \quad \forall n \geq n_{0} .
$$

Using (24), there is a sequence of neighborhoods $\left\{N_{\delta_{n}}(\theta)\right\}_{n \geq n}$ such that $[z-(r(k+l) / 1-[r(k+l)])]$ $(k+l)^{n} z_{0}+N_{\delta_{n}}(\theta) \subset C$, using (23) gives

$$
\begin{aligned}
& {\left[(k+l)^{n}\left[\frac{r(k+l)}{1-[r(k+l)]}\right] z_{0}-q\left(u_{n}, u_{m}\right)+q\left(v_{n}, v_{m}\right)\right]} \\
& +\left[z-\frac{r(k+l)}{1-[r(k+l)]}\right](k+l)^{n} z_{0}+N_{\delta_{n}}(\theta) \\
& \quad \subset C+C \subset C, \quad \forall n \geq n_{0} .
\end{aligned}
$$

This means

$$
\left[z-q\left(u_{n}, u_{m}\right)+q\left(v_{n}, v_{m}\right)\right]+N_{\delta_{n}}(\theta) \subset C, \quad \forall n \geq n_{0} .
$$

Consequently, $\left[z-q\left(u_{n}, u_{m}\right)+q\left(v_{n}, v_{m}\right)\right] \in \operatorname{Int}(C)$ for every $n \geq n_{0}$, and we have

$$
q\left(u_{n}, u_{m}\right)+q\left(v_{n}, v_{m}\right) \ll z, \quad \forall n \geq n_{0} .
$$

Since $\quad n \leq m, \quad q\left(u_{n}, u_{m}\right) \leq q\left(u_{n}, u_{m}\right)+q\left(v_{n}, v_{m}\right)$, and $q\left(v_{n}, v_{m}\right) \leq q\left(u_{n}, u_{m}\right)+q\left(v_{n}, v_{m}\right)$, we similarly conclude that

$$
\begin{aligned}
& q\left(u_{n}, u_{m}\right) \ll z, \quad \forall n, m \geq n_{0}, \\
& q\left(v_{n}, v_{m}\right) \ll z, \quad \forall n, m \geq n_{0} .
\end{aligned}
$$

The two inequalities (28) and (29) prove that the two sequences $\left\{u_{n}\right\}_{n \in \mathbb{N}}$ and $\left\{v_{n}\right\}_{n \in \mathbb{N}}$ are Cauchy sequences in $(X, C, q)$. Since $(X, C, q)$ is complete $b$ - cone metric space, there are two limits. Let $f \geq 1$, we have $\theta \ll[(1 / r[k+l+1] f)] z$. Then,

$$
\begin{array}{ll}
\forall \theta \ll z \exists n_{1} \in \mathbb{N} \text { such that } q\left(u_{n}, w_{0}\right) \ll\left[\frac{1}{r[k+l+1] f}\right] z, & \forall n \geq n_{1}, \\
\forall \theta \ll z \exists n_{2} \in \mathbb{N} \text { such that } q\left(v_{n}, z_{0}\right) \ll\left[\frac{1}{r[k+l+1] f}\right] z, & \forall n \geq n_{2} .
\end{array}
$$

Take $N_{0}=\max \left\{n_{1}, n_{2}\right\}$, and we have

$$
\begin{aligned}
q & \left(T\left(w_{0}, z_{0}\right), w_{0}\right) \leq r\left[q\left(T\left(w_{0}, z_{0}\right), u_{N_{0}+1}\right)+q\left(u_{N_{0}+1}, w_{0}\right)\right] \\
= & r\left[q\left(T\left(w_{0}, z_{0}\right), T\left(u_{N_{0}}, v_{N_{0}}\right)\right)+q\left(u_{N_{0}+1}, w_{0}\right)\right] \\
= & r\left[k q\left(w_{0}, u_{N_{0}}\right)+l q\left(z_{0}, v_{N_{0}}\right)\right]+r q\left(u_{N_{0}+1}, w_{0}\right) \\
= & r\left[k\left[\frac{1}{r[k+l+1] f}\right] z+l\left[\frac{1}{r[k+l+1] f}\right] z\right] \\
& +r\left[\frac{1}{r[k+l+1] f}\right] z \\
= & r[k+l+1]\left[\frac{1}{r[k+l+1] f}\right] z=\left[\frac{1}{f}\right] z .
\end{aligned}
$$

Since $f \geq 1$ is an arbitrary number, we have $q\left(T\left(w_{0}, z_{0}\right), w_{0}\right)=\theta$, and hence, $T\left(w_{0}, z_{0}\right)=w_{0}$, and similarly, $T\left(z_{0}, w_{0}\right)=z_{0}$, meaning that $\left(w_{0}, z_{0}\right)$ is a coupled fixed point of $T$. Finally, we show that such a coupled fixed point is unique. Contrarily, suppose that $\left(u_{1}, v_{1}\right)$ is another coupled fixed point. Then, we have

$$
\begin{aligned}
q\left(w_{0}, u_{1}\right) & =q\left(T\left(w_{0}, z_{0}\right), T\left(u_{1}, v_{1}\right)\right) \leq k q\left(w_{0}, u_{1}\right)+l q\left(z_{0}, v_{1}\right), \\
q\left(z_{0}, v_{1}\right) & =q\left(T\left(z_{0}, w_{0}\right), T\left(v_{1}, u_{1}\right)\right) \leq k q\left(z_{0}, v_{1}\right)+l q\left(w_{0}, u_{1}\right) .
\end{aligned}
$$

Adding, we obtain

$$
q\left(w_{0}, u_{1}\right)+q\left(z_{0}, v_{1}\right) \leq[k+l]\left[q\left(w_{0}, u_{1}\right)+q\left(z_{0}, v_{1}\right)\right] .
$$

Since $[k+l]<1$, we see that $q\left(w_{0}, u_{1}\right)+q\left(z_{0}, v_{1}\right)=\theta$; hence, $q\left(w_{0}, u_{1}\right)=\theta$ and $q\left(z_{0}, v_{1}\right)=\theta$ meaning that $\left(w_{0}, z_{0}\right)=\left(u_{1}, v_{1}\right)$ and the proof of the theorem is completed.

The following corollary proves Theorem 1 of Sabetghadam et al.

Corollary 1. Let $(X, C, q)$ be a complete cone metric space. Suppose that the mapping $T: X \times X \longrightarrow X$ satisfies the following contractive condition for all $x, y, u, v \in X$ :

$$
q(T(x, y), T(u, v)) \leq k q(x, u)+l q(y, v),
$$

where $k$ and $l$ are nonnegative constants with $k+l<1$. Then, $T$ has a unique coupled fixed point.

Proof. Using Theorem 2 with $r=1$ completes the proof.

We also have the following.

Corollary 2. Let $(X, C, q)$ be a complete cone metric space. Suppose that the mapping $T: X \times X \longrightarrow X$ satisfies the following contractive condition for all $x, y, u, v \in X$ : 


$$
q(T(x, y), T(u, v)) \leq t[q(x, u)+q(y, v)],
$$

where $t$ is a nonnegative constant with $t<(1 / 2)$. Then, T has a unique coupled fixed point.

Proof. Using Corollary 1 with $l=k<(1 / 2)$, we obtain the following.

Corollary 3. Let $(X, C, q)$ be a complete b-cone metric space. Suppose that the mapping $T: X \times X \longrightarrow X$ satisfies the following contractive condition for all $x, y, u, v \in X$ :

$$
q(T(x, y), T(u, v)) \leq t[q(x, u)+q(y, v)],
$$

where $t$ is nonnegative constant with $r t<(1 / 2)$. Then, $T$ has a unique coupled fixed point.

Proof. Using Theorem 2 with $k=l$ completes the proof.

Theorem 3. Let $(X, C, q)$ be a complete b-cone metric space. Suppose that the mapping $T: X \times X \longrightarrow X$ satisfies the following contractive condition for all $x, y, u, v \in X$ :

$$
q(T(x, y), T(u, v)) \preceq a q(x, u)+b q(T(x, y), x)+c q(T(u, v), u), \quad \forall x, y, u, v \in X
$$

where $a, b, c \in \mathbb{R}$ are nonnegative constants with $r[a+b+c]<1$. Then, $T$ has a unique coupled fixed point.

Proof. Construct the two sequences $\left\{u_{n}\right\}_{n \in \mathbb{N}}$ and $\left\{v_{n}\right\}_{n \in \mathbb{N}}$ as in Theorem 2. Then, by (37), we have

$$
\begin{aligned}
& q\left(u_{n+1}, u_{n}\right)=q\left(T\left(u_{n}, v_{n}\right), T\left(u_{n-1}, v_{n-1}\right)\right) \\
& \preceq a q\left(u_{n}, u_{n-1}\right)+b q\left(T\left(u_{n}, v_{n}\right), u_{n}\right)+c q\left(T\left(u_{n-1}, v_{n-1}\right), u_{n-1}\right) \\
& \preceq a q\left(u_{n}, u_{n-1}\right)+b q\left(u_{n+1}, u_{n}\right)+c q\left(u_{n}, u_{n-1}\right) \\
& \preceq[a+c] q\left(u_{n}, u_{n-1}\right)+b q\left(u_{n+1}, u_{n}\right) .
\end{aligned}
$$

Consequently,

$$
q\left(u_{n+1}, u_{n}\right) \preceq\left[\frac{a+c}{1-b}\right] q\left(u_{n}, u_{n-1}\right) .
$$

Similarly,

$$
q\left(v_{n+1}, v_{n}\right) \preceq\left[\frac{a+c}{1-b}\right] q\left(v_{n}, v_{n-1}\right) .
$$

We have $r[(a+c / 1-b)]<1$; then, the two sequences $\left\{u_{n}\right\}_{n \in \mathbb{N}}$ and $\left\{v_{n}\right\}_{n \in \mathbb{N}}$ are Cauchy sequences, and the rest of the proof is similar to the proof of Theorem 2 .

Corollary 4. Let $(X, C, q)$ be a complete $b$-cone metric space. Suppose that the mapping $T: X \times X \longrightarrow X$ satisfies the following contractive condition for all $x, y, u, v \in X$ :

$$
\begin{aligned}
& q(T(x, y), T(u, v)) \preceq t[q(T(x, y), x)+q(T(u, v), u)], \\
& \quad \forall x, y, u, v \in X
\end{aligned}
$$

where $t \in \mathbb{R}$ is nonnegative constants with $r t<(1 / 2)$. Then, $T$ has a unique coupled fixed point.

The following is a coupled fixed point theorem of contraction type of mappings in $b$ - $\Theta$-cone metric spaces.

Theorem 4. Let $\left(X, C, d_{\Theta}\right)$ be a complete $b$ - $\Theta$-cone metric space. Suppose that the mapping T: $X \times X \longrightarrow X$ satisfies the following contractive condition for all $x, y, u, v \in X$ :

$$
d_{\Theta}(T(x, y), T(u, v)) \preceq k d_{\Theta}(x, u)+l d_{\Theta}(y, v),
$$

where $k$ and $l$ are nonnegative constants with $k+l<1$. Then, $T$ has a unique coupled fixed point.

Proof. Construct the two sequences $\left\{u_{n}\right\}_{n \in \mathbb{N}}$ and $\left\{v_{n}\right\}_{n \in \mathbb{N}}$ in $X$ and the sequence $\left\{z_{n}\right\}_{n \in \mathbb{N}}$ in $C$ as in Theorem 2 . Then, by (42), we have

$$
\begin{aligned}
& d_{\Theta}\left(u_{n+1}, u_{n}\right) \preceq k d_{\Theta}\left(u_{n}, u_{n-1}\right)+l d_{\Theta}\left(v_{n}, v_{n-1}\right) \\
& \prec(k+l)\left[d_{\Theta}\left(u_{n}, u_{n-1}\right)+d_{\Theta}\left(v_{n}, v_{n-1}\right)\right], \\
& \quad d_{\Theta}\left(v_{n+1}, v_{n}\right) \preceq k d_{\Theta}\left(v_{n}, v_{n-1}\right)+l d_{\Theta}\left(u_{n}, u_{n-1}\right) \\
& \preceq(k+l)\left[d_{\Theta}\left(u_{n}, u_{n-1}\right)+d_{\Theta}\left(v_{n}, v_{n-1}\right)\right], \\
& \quad \theta \preceq z_{n} \preceq(k+l) z_{n-1} \preceq(k+l)^{2} z_{n-2} \preceq \cdots \preceq(k+l)^{n} z_{0} .
\end{aligned}
$$

The last inequalities give

$$
\begin{aligned}
& d_{\Theta}\left(u_{n+1}, u_{n}\right) \preceq(k+l) z_{n} \preceq(k+l)^{n+1} z_{0}, \\
& d_{\Theta}\left(v_{n+1}, v_{n}\right) \preceq(k+l) z_{n} \preceq(k+l)^{n+1} z_{0} .
\end{aligned}
$$

If $z_{0}=\theta$, then $\left(x_{0}, y_{0}\right)$ is coupled fixed of $T$; therefore, we continue by letting $\theta<z_{0}$. Now, let $\theta \ll z, z \in \operatorname{Int}(C)$. Then, there is a neighborhood of $z$ with some radius $\delta>0$ say $z+N_{\delta}(\theta)$ such that $z+N_{\delta}(\theta) \subset C$, and for this $\delta>0$, there is a natural number $n_{0}$ such that $(k+l)^{n+1}<\delta\left[\left(1 /\left\|z_{0}\right\|\right)\right]$ for every $n \geq n_{0}$; hence, $\left\|z_{0}\right\|(k+l)^{n+1}<\delta$ for every $n \geq n_{0}$; consequently, $\left\|(k+l)^{n+1} z_{0}\right\|<\delta$ for every $n \geq n_{0}$. This proves that $\pm(k+l)^{n+1} z_{0} \in N_{\delta}(\theta)$ for every $n \geq n_{0}$. Therefore,

$$
(k+l)^{n+1} z_{0} \ll z, \quad \forall n \geq n_{0} .
$$

Using (46) with both (44) and (45), we have

$$
\begin{aligned}
& d_{\Theta}\left(u_{n+1}, u_{n}\right) \ll z, \quad \forall n \geq n_{0}, \\
& d_{\Theta}\left(v_{n+1}, v_{n}\right) \ll z, \quad \forall n \geq n_{0} .
\end{aligned}
$$

Now, we are going to show that the two sequences $\left\{u_{n}\right\}_{n \in \mathbb{N}}$ and $\left\{v_{n}\right\}_{n \in \mathbb{N}}$ are Cauchy sequences; for this reason, suppose that one of them is not Cauchy, say $\left\{u_{n}\right\}_{n \in \mathbb{N}}$. Then, 
there exists $\bar{z} \in \operatorname{Im}(\Theta), \theta<\bar{z}$ and sequences of natural numbers $\left\{i_{n}\right\}_{n \in N}$ and $\left\{j_{n}\right\}_{n \in N}$ such that for any $i_{n}>j_{n}>n$,

$$
\begin{array}{r}
\bar{z} \preceq d_{\Theta}\left(u_{i_{n}}, u_{j_{n}}\right), \\
d_{\Theta}\left(u_{i_{n}-1}, u_{j_{n}}\right)<\bar{z} .
\end{array}
$$

Let $f \in \mathbb{R}^{+}$be such that $r \Theta(f \bar{z}, \bar{z})<\bar{z}$; using (47), for such $\bar{z}$, there is $n_{0} \in \mathbb{N}$ such that $d_{\Theta}\left(u_{n+1}, u_{n}\right) \ll f \bar{z}$ for every $n \geq n_{0}$; in particular, we have $d_{\Theta}\left(u_{i_{n}}, u_{i_{n}-1}\right) \ll f \bar{z}$ for every $i_{n} \geq n_{0}$; hence, we have the following contradiction:

$$
\begin{aligned}
\bar{z} & \leq d_{\Theta}\left(u_{i_{n}}, u_{j_{n}}\right) \preceq r \Theta\left(d_{\Theta}\left(u_{i_{n}}, u_{i_{n}-1}\right), d_{\Theta}\left(u_{i_{n}-1}, u_{j_{n}}\right)\right) \\
& <r \Theta\left(d_{\Theta}\left(u_{i_{n}}, u_{i_{n}-1}\right), \bar{z}\right) \preceq r \Theta(f \bar{z}, \bar{z})<\bar{z} .
\end{aligned}
$$

Hence, the two sequences are Cauchy sequences. Since $\left(X, C, d_{\Theta}\right)$ is complete, there are two limits for both of them say $u_{0}$ and $v_{0}$, respectively:

$$
\begin{aligned}
& u_{n} \longrightarrow \stackrel{d_{\Theta}}{\longrightarrow} \infty u_{0}, \\
& v_{n} \longrightarrow \stackrel{d_{\Theta}}{\longrightarrow} \infty v_{0} \text {. }
\end{aligned}
$$

We show that $\left(u_{0}, v_{0}\right)$ is coupled fixed point of $T$. Using the properties of $\Theta$, we see that

$$
\begin{array}{r}
d_{\Theta}\left(T\left(u_{0}, v_{0}\right), u_{0}\right) \preceq r \Theta\left(d_{\Theta}\left(T\left(u_{0}, v_{0}\right), u_{n}\right), d_{\Theta}\left(u_{n}, u_{0}\right)\right) \\
\preceq r \Theta\left(d_{\Theta}\left(T\left(u_{0}, v_{0}\right), T\left(u_{n-1}, v_{n-1}\right)\right), d_{\Theta}\left(u_{n}, u_{0}\right)\right) \\
\preceq r \Theta\left(\left(k d_{\Theta}\left(u_{0}, u_{n-1}\right)+l d_{\Theta}\left(v_{0}, v_{n-1}\right)\right), d_{\Theta}\left(u_{n}, u_{0}\right)\right) \\
\longrightarrow{ }_{n \longrightarrow \infty} r \Theta((k \theta+l \theta), \theta)=r \Theta(\theta, \theta)=r \theta=\theta .
\end{array}
$$

Consequently, $d_{\Theta}\left(T\left(u_{0}, v_{0}\right), u_{0}\right)=\theta$, meaning that $T\left(u_{0}, v_{0}\right)=u_{0}$; similarly, $T\left(v_{0}, u_{0}\right)=v_{0}$. The uniqueness of such a coupled fixed point is as before; clearly, the coupled fixed point is unique.

Corollary 5. Let $\left(X, C, d_{\Theta}\right)$ be a complete theta cone metric space. Suppose that the mapping T: $X \times X \longrightarrow X$ satisfies the following contractive condition for all $x, y, u, v \in X$ :

$$
d_{\Theta}(T(x, y), T(u, v)) \preceq k d_{\Theta}(x, u)+l d_{\Theta}(y, v),
$$

where $k$ and $l$ are nonnegative constants with $k+l<1$. Then, $T$ has a unique coupled fixed point.

As in Theorem 3, we have the following.

Corollary 6. Let $\left(X, C, d_{\Theta}\right)$ be a complete $\Theta$-cone metric space. Suppose that the mapping T: $X \times X \longrightarrow X$ satisfies the following contractive condition for all $x, y, u, v \in X$ :

$$
\begin{aligned}
& d_{\Theta}(T(x, y), T(u, v)) \preceq a d_{\Theta}(x, u)+b d_{\Theta}(T(x, y), x) \\
& +c d_{\Theta}(T(u, v), u), \quad \forall x, y, u, v \in X,
\end{aligned}
$$

where $a, b, c \in \mathbb{R}$ are nonnegative constants with $a+b+c<1$. Then, $T$ has a unique coupled fixed point.

Corollary 7. Let $\left(X, C, d_{\Theta}\right)$ be a complete $b$ - $\Theta$-cone metric space. Suppose that the mapping T: $X \times X \longrightarrow X$ satisfies the following contractive condition for all $x, y, u, v \in X$ :

$$
\begin{aligned}
& q(T(x, y), T(u, v)) \preceq t\left[d_{\Theta}(T(x, y), x)+d_{\Theta}(T(u, v), u)\right] \\
& \quad \forall x, y, u, v \in X
\end{aligned}
$$

where $t \in \mathbb{R}$ is nonnegative constants with $t<(1 / 2)$. Then, $T$ has a unique coupled fixed point.

\section{Conclusion}

The results of this paper prove the existence of a unique coupled fixed point of some well-known type contraction of mappings but are defined on complete $b$-cone and $b$-theta cone metric spaces; consequently, it extends and generalizes some previous coupled fixed point theorems.

\section{Data Availability}

No data were used to support this study.

\section{Conflicts of Interest}

The author has no conflicts of interest.

\section{Authors' Contributions}

The sole author contributed $100 \%$ to the article. The author read and approved the final manuscript.

\section{Acknowledgments}

Sincere thanks go to the anonymous referees and editors for their valuable comments and kind collaboration with this work to appear.

\section{References}

[1] L. G. Huang and X. Zhang, "Cone metric spaces and fixed point theorems of contractive mappings," Journal of Mathematical Analysis and Applications, vol. 332, no. 2, pp. 1468-1476, 2007.

[2] Sh. Rezapour and R. Hamlbarani, "Some notes on the paper Cone metric spaces and fixed point theorems of contractive mappings," Journal of Mathematical Analysis and Applications, vol. 345, no. 2, Article ID 719724, 2008.

[3] R. H. Haghi and Sh. Rezapour, "Fixed points of multifunctions on regular cone metric spaces," Expositiones Mathematicae. in Numerical Functional Analysis and Optimization, vol. 30, no. 7, pp. 825-832, 2009.

[4] W. S. Du, "A note on cone metric fixed point theory and its equivalence," Nonlinear Analysis: Theory, Methods and Applications, vol. 72, no. 5, pp. 2259-2261, 2010.

[5] H. Liu and S. Xu, "Cone metric spaces with Banach algebras and fixed point theorems of generalized Lipschitz mappings," Fixed Point Theory Application, 2013, 2013, Article ID 320.

[6] S. Xu and S. Radenovic, "Fixed point theorems of generalized Lipschitz mappings on cone metric spaces over Banach algebras without assumption of normality," Fixed Point Theory and Applications, p. 102, 2014.

[7] H. P. Huang and S. Radenovic, "Some fixed point results of generalised Lipschitz mappings on cone b-metric spaces over Banach algebras," Journal of Computational Analysis and Applications, vol. 20, pp. 566-583, 2016. 
[8] N. Sharma, "Fixed point theorem in cone B metric spaces using contractive mappings," Global Journal of Pure and Applied Mathematics, vol. 13, no. 7, pp. 2997-3004, 2017.

[9] S. M. A. Abou Bakr, "A study on common fixed point of joint $(\mathrm{A}, \mathrm{B})$ generalized cyclic $\phi-a b c$ weak nonexpansive mappings and generalized cyclic $a b c ; r$ contractions in quasi metric spaces," Abstract and Applied Analysis, vol. 2020, Article ID 9427267, 10 pages, 2020.

[10] S. M. A. Abou Bakr, "Theta cone metric spaces and some fixed point theorems," Journal of Mathematics, vol. 2020, Article ID 8895568, 7 pages, 2020.

[11] D. Guo and V. Lakshmikantham, "Coupled fixed points of nonlinear operators with applications," Nonlinear Analysis: Theory, Methods and Applications, vol. 11, no. 5, pp. 623-632, 1987.

[12] T. G. Bhaskar and V. Lakshmikantham, "Fixed point theorems in partially ordered metric spaces and applications," Nonlinear Analysis: Theory, Methods and Applications, vol. 65, no. 7, pp. 1379-1393, 2006.

[13] F. Sabetghadam, H. P. Masiha, H. P. Masiha, and A. H. Sanatpour, "Some coupled fixed point theorems in cone metric spaces," Fixed Point Theory and Applications, vol. 2009, Article ID 125426, 9 pages, 2009.

[14] M. A. Khamsi, "Remarks on cone metric spaces and fixed point theorems of contractive mappings," Fixed Point Theory and Applications, vol. 2010, Article ID 315398, 7 pages, 2010.

[15] N. V. Luong, N. X. Thuan, and K. P. R. Rao, "Remarks on coupled fixed point theorems in cone metric spaces," Matematiqki Vesnik, vol. 65, p. 1, Article ID 122136, 2013.

[16] S. M. A. Abou Bakr, "Coupled fixed-point theorems in thetacone-metric spaces," Journal of Mathematics, vol. 2021, Article ID 6617738, 7 pages, 2021.

[17] S. Mohamed Ali., "Fixed point theorems of some type contraction mappings," Journal of Nonlinear and Convex Analysis, vol. 14, no. 2, Yokohama Publishing Corporation, [ISSN 1880-5221], , pp. 331-342, 2013.

[18] E. M. Elshobaky, S. M. A. Abou Bakr, and S. A. Montaser, "Generalization of Banach contraction principle in two directions," The International Journal of Mathematics and Statistics USA,JMSSP, vol. 3, no. No. 3, pp. 112-115, 2007, [ISSN 1549-3644]. 